\title{
Reported National Level Stock-Outs of Home-Based Records-A Quiet Problem for Immunization Programmes That Needs Attention
}

\author{
David W. Brown'1, Marta Gacic-Dobo² \\ ${ }^{1}$ Brown Consulting Group International, LLC, Charlotte, USA \\ ${ }^{2}$ World Health Organization, Geneva, Switzerland \\ Email: gacicdobom@who.int
}

How to cite this paper: Brown, D.W. and Gacic-Dobo, M. (2017) Reported National Level Stock-Outs of Home-Based Records-A Quiet Problem for Immunization Programmes That Needs Attention. World Journal of Vaccines, 7, 1-10.

http://dx.doi.org/10.4236/wjv.2017.71001

Received: November 16, 2016

Accepted: January 7, 2017

Published: January 10, 2017

Copyright $\odot 2017$ by authors and Scientific Research Publishing Inc. This work is licensed under the Creative Commons Attribution International License (CC BY 4.0).

http://creativecommons.org/licenses/by/4.0/

\begin{abstract}
Home-based records (HBRs) are an important tool for recording and communicating within primary healthcare service delivery. Unfortunately, HBRs are currently unable to fulfil their intended purpose in many communities either because the HBR is not functionally well-designed to serve its objectives, not made available, not fully adopted and/or not appropriately utilized by caregivers and/or health workers. This brief report describes the occurrence of nationally reported HBR stock-outs and HBR financing patterns during 2014 and 2015 across 195 countries reporting immunization system performance data to the World Health Organization and United Nations Children's Fund. National level HBR stock-outs were reported by 19 and 22 countries during 2014 and 2015, respectively, with eleven countries reporting stock-outs during both 2014 and 2015. During 2015, 12 of the 22 countries reporting HBR stock-outs were from the African Region and two-thirds of the countries were Gavi-eligible. Information on HBR stock-outs was either not available or not reported by 66 countries ( 19 were Gavi-eligible) for 2014 and 53 (11 were Gavi-eligible) countries for 2015. Among the 22 countries reporting HBR stock-outs in 2015, 12 (54\%) countries reported a single HBR financing source, and nine (41\%) countries reported more than one source for HBR financing. The occurrence of HBR stock-outs remains a concern, particularly in Gavi-eligible countries introducing new vaccines where dedicated funding is received for revising and printing new recording tools, including HBRs. Additional attention is needed to understand the root causes for stock-outs and identify solutions to ensure a well-designed, durable HBR is readily available in the right quantity, in the right place at the right time in all countries.
\end{abstract}




\section{Keywords}

Home-Based Record, Personal Health Record, Supply, Stock-Out, Recording, Monitoring

\section{Introduction}

Home-based personal health records-from this point forward referred to as home-based records (HBRs) - used to record immunization and other primary care services received (including the date-of-service) by individuals are an important tool within primary healthcare service delivery that encourages a partnership in the care of the child between the healthcare worker and the caregiver. Within primary healthcare service delivery, HBRs are an important recording tool and information resource (i) to enhance health professionals' ability to make appropriate clinical decisions (e.g., which vaccinations have been received already and which vaccinations remain outstanding) and improve continuity of care across providers in the absence of other health records, (ii) to empower individuals or their caregivers as a vehicle for health education about which primary healthcare services have been received and those which remain outstanding as well as (iii) to support public health monitoring efforts (i.e., through population-based vaccination coverage surveys). Unfortunately, HBRs are currently unable to fulfil their intended purpose in many communities either because the HBR is not functionally well-designed to serve the needs above, not made available, not fully adopted and/or not appropriately utilized by caregivers and/or health workers.

The challenges of HBR availability have previously been highlighted with nearly one-quarter of respondent countries reporting national level HBR stock-outs during 2013 [1], meaning that the HBR was not printed in sufficient quantities for all newborns and their caregivers to receive a document that they are rightly due as part of national promises made in the Convention on the Rights of the Child (Articles 3 and 24) [2] to protect children's health through primary healthcare and engagement of caregivers in making decisions about the healthcare (and vaccination needs) of their child. This report describes an update on reported HBR stock-outs by countries during 2014 and 2015 (reported during 2015 and 2016, respectively). We also describe reported HBR financing patterns during the same period.

\section{Methods}

\subsection{Data Source}

Since 1998, the World Health Organization (WHO) and United Nations Children's Fund (UNICEF) have jointly collected national immunization system performance data using a standardized data collection form, the Joint Reporting Form on Immunization (JRF). Since its inception, the JRF, which was imple- 
mented to avoid the publication and dissemination of discrepant immunization system performance data and to take advantage of operational efficiencies within each agency, has evolved from a limited set of information collected using a paper-based form to one based in MS Excel (Microsoft Corporation, Redmond, Washington) capturing a wide range of domains of standard performance, planning, financing and quality indicators (Box 1). The information collected in the JRF serves as a critical resource for tracking implementation of the Global Vaccine Action Plan (GVAP), endorsed by all WHO Member States at the World Health Assembly (WHA) in 2012 [3], and the Regional Vaccine Action Plans (RVAPs). These initiatives serve as key frameworks to guide immunization strategies at global and regional levels, and progress reports on GVAP, developed using data reported by countries through the JRF, are presented to the WHA each year. Other uses of the immunization system performance data include monitoring the health situation and assessing health trends, one of the WHO and UNICEF core functions; monitoring progress towards the Millennium Development Goals and Sustainable Development Goals; contributing to the WHO and UNICEF estimation of national immunization coverage process and informing vaccine-preventable disease burden estimates.

The form which is coordinated and maintained by WHO Headquarters (Geneva, Switzerland) and UNICEF Headquarters (United Nations, New York), is distributed via e-mail to national immunization programmes or the immuniza-

Box 1. Information collected from national immunization programmes through the WHO-UNICEF joint reporting form on immunization.

- Contact details for national immunization programme staff as well as WHO and UNICEF country office immunization focal points;

- $\quad$ Reported cases of selected vaccine preventable diseases for which there is no other free-standing reporting system (e.g., polio) and general information on surveillance systems;

- Updates to national immunization schedules;

- Sources of vaccines and supplies;

- School based immunization activities;

- Administrative data system derived immunization coverage;

- Official government immunization coverage estimate;

- Immunization system planning and management indicators;

- National immunization advisory mechanism indicators;

- Aggregate district coverage for routine immunization for third dose of diphtheria and tetanus toxoid with pertussis vaccine and first dose of measles containing vaccine;

- Vaccine and supply stock-out information;

- Vaccine safety indicators;

- Home-based records;

- Immunization financing data; and,

- Supplementary immunization activities completed and planned to name a few. 
tion focal person in the Ministry of Health in all Member States of the WHA, the State of Palestine (a non-voting observer to the WHA) as well as several Western Pacific territories in January/February each year by WHO and UNICEF regional and country offices. Once completed by national immunization programme staff in collaboration with WHO and UNICEF country office counterparts, the forms are returned to $\mathrm{WHO} / \mathrm{Geneva}$ and/or UNICEF/New York via e-mail attachment along the same reporting channels by which the forms were distributed. National authorities are currently requested to return data submissions by mid-April; prior to 2009 data submissions were requested by mid-May. For the most recent reporting period in 2016 (data collected for 2015), 96\% (187/195, inclusive of the State of Palestine) of countries reported data on the JRF, an improvement from $68 \%(131 / 192)$ of countries in 2000.

The immunization system performance data are collected for a calendar year, January through December, and countries may update prior years' data at any time through written communication to WHO and/or UNICEF. After forms are received by WHO and UNICEF, data are extracted, reviewed for completeness and consistency and queries are sent to countries to clarify absent information and inconsistencies. The nationally reported immunization performance data are then made publicly available on the WHO website (www.who.int/immunization/monitoring_surveillance) and updated on the website twice per year (June, December).

\subsection{Questions on Home-Based Record Stock-Outs and Financing}

During 2014 and 2015, the JRF included the following question for countries to respond: "Was there a stock-out of home-based vaccination records for children (no remaining home-based records for any period of time) at the national level during 201(4/5)?" Countries were also asked, "Which organization is responsible for financing the home-based records for children in your country?" A multiselect multiple choice response category was provided allowing the respondent to select any combination of the following: (i) immunization programme or Ministry of Health (EPI/MOH), (ii) other government agency and (iii) other with request for additional explanatory detail.

Results were tallied at the global level and by WHO operational region (see www.who.int/about/structure) and eligibility for Phase 2 financial support from Gavi, the Vaccine Alliance (see www.gavi.org).

\section{Results}

A total of 19 countries reported national level HBR stock-outs during 2014, and 22 countries reported HBR stock-outs during 2015 (Table 1). Eleven countries reported national level HBR stock-outs during both 2014 and 2015 (Annex). Among the 19 countries reporting HBR stock-outs in 2014, 11 countries were from the WHO African Region, four from the Region of the Americas, three from the Western Pacific Region and one from the Eastern Mediterranean Region (Annex). During 2015, 12 of the 22 countries reporting HBR stock-outs 
Table 1. Frequency of countries reporting national level home-based record (HBR) stockouts during 2014 and 2015 by WHO operational region and eligibility for Phase 2 assistance from Gavi, the Vaccine Alliance.

\begin{tabular}{lcc}
\hline Reported HBR stock-out & 2014 & 2015 \\
\hline Yes & $19(10 \%)$ & $22(11 \%)$ \\
African Region $(\mathrm{n}=47$ countries) & 11 & 12 \\
Region of the Americas $(\mathrm{n}=35)$ & 4 & 3 \\
Eastern Mediterranean Region $\left(\mathrm{n}=22^{\mathrm{a}}\right)$ & 1 & 1 \\
European Region $(\mathrm{n}=53)$ & 0 & 1 \\
South-East Asia Region $(\mathrm{n}=11)$ & 0 & 5 \\
Western Pacific Region $(\mathrm{n}=27)$ & 3 & 15 \\
Gavi-eligible $(\mathrm{n}=73)^{\mathrm{c}}$ & 10 & $118(61 \%)$ \\
No & $108(55 \%)$ & $53(27 \%)$ \\
No Report/No Data & $66(34 \%)$ & $2(1 \%)$ \\
HBR not used in system & $\mathrm{b}$ & 195 \\
Total no. countries & 195 & $1 \%)$ \\
\hline
\end{tabular}

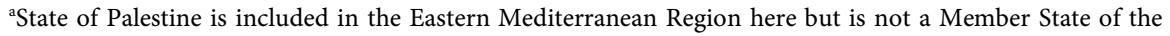
World Health Assembly as of this writing and not officially part of the WHO Regional Office for the Eastern Mediterranean; ${ }^{b}$ Based on a prior report to the World Health Organization [1]; 'Countries eligible for Phase 2 support from Gavi, the Vaccine Alliance can be found online at www.gavi.org.

were from the African Region, five from the Western Pacific Region, three from the Region of the Americas and one each from the Eastern Mediterranean and South-East Asia Regions (Annex). Seven of the 11 countries reporting HBR stock-outs during 2014 and 2015 were from Africa, two from the Region of the Americas and one each from the Eastern Mediterranean and Western Pacific Regions. Among the countries reporting HBR stock-outs in 2014, nearly half were Gavi-eligible; in 2015, two-thirds of the countries reporting stock-outs were Gavi-eligible.

Information on HBR stock-outs was either not available or not reported by 66 countries (19 were Gavi-eligible) for 2014 and 53 (11 were Gavi-eligible) countries for 2015. We are aware of two countries, Belarus and Norway that notified the WHO in 2014 that they do not utilized HBRs in their immunization system. Of the 53 countries without a report on HBR stock-outs for 2015 (Norway and Belarus reported during 2013 that they do not use home-based records [1]), five countries were from the African Region, five countries from the Region of the Americas, seven countries from the Eastern Mediterranean Region, 26 countries from the European Region, two countries from South-East Asia and eight countries from the Western Pacific Region.

Overall, 80 countries reported that EPI/MOH was solely responsible for HBR financing, five countries noted that another government agency was solely responsible and 11 countries noted that other non-governmental partners were solely responsible for HBR financing. Among the remaining 99 countries, 40 countries reported more than one source of HBR financing (59 countries either 
chose not to report information on HBR financing or had no data to report). Among the 22 countries reporting HBR stock-outs in 2015, 12 (54\%) reported a single HBR financing source (EPI/MOH: 9; other non-government partners: 3), and nine (41\%) countries reported more than one source for HBR financing. Among the 118 countries not reporting a HBR stock-out during 2015, 69 (58\%) countries reported a single source for HBR financing (EPI/MOH: 60; other government: 3 ) and 24 (20\%) countries reported more than one source for HBR financing. Of the 38 countries noting non-governmental HBR financing support during 2015, 21 (55\%) countries noted UNICEF, either alone or in combination with other partners; no other partner was noted as frequently (WHO was mentioned by 10 countries). Similar HBR financing results were observed for 2014 .

\section{Comment}

Of the more than 140.2 million estimated births during 2015, roughly $9 \%$ (estimated 12.4 million) were born in 22 countries reporting a national level HBR stock-out. Two-thirds (or 8.3 million) of these children resided in one of 12 countries in the WHO African Region, and more than half (4.8 million) of these children resided in the Democratic Republic of Congo or Kenya. Other countries with large $(>500,000)$ birth cohorts reporting national level stock-outs included the Philippines, Ghana, Cameroon, Malawi, Chad and Venezuela. Taking into consideration results from an assessment of national level HBR stock-outs in 2013 collected using a similar approach [1], four countries (Chad, Democratic Republic of the Congo, Guinea-Bissau, Venezuela) have reported HBR stock-outs for 2013, 2014 and 2015.

The presence of HBR stock-outs observed here for Gavi-eligible countries introducing new vaccines into the routine immunization schedule is particularly troubling given that these countries receive dedicated funding for revising and printing new recording tools, including HBRs, as part of the introduction grant. For this reason, it is seemingly worthwhile to further explore the system breakdowns which led to HBR stock-outs in the countries that introduced either pneumococcal conjugate vaccine (PCV) or rotavirus vaccine (rota) within two years of the reported HBR stock-out, including: Burundi (rota, 2013), Cambodia (PCV, 2015), Cameroon (rotavirus vaccine, 2014), Democratic Republic of the Congo (PCV, 2013), Guinea-Bissau (PCV, 2015), Kenya (rota, 2014), Kiribati (rota, 2015), Laos (PCV, 2013), Mauritania (PCV, 2013; rota, 2014), Niger (PCV, 2014; rota, 2014), Solomon Islands (PCV, 2015) and Togo (PCV, 2014; rota, 2014).

Presumably HBR stock-outs in these countries would be related to poor planning and lacking attention to the time necessary to update and print new HBRs (and other recording tools such as registers and tally sheets) as part of the new vaccine introduction planning process. Reports on lessons learned during new vaccine introduction and the post-introduction evaluation reports highlight lacking immunization programme control over the HBR and recording tool updates as a bottleneck leading to delays in form updates and distribution [4]. In 
most instances, proper prior planning and communication with relevant departments involved in the HBR and recording tool update, printing and distribution continuum in advance of the new vaccine introduction are the recommended solution. Unfortunately, because of overall lacking attention to recording and reporting needs and practices, too often these recommendations go unheeded and problematic experiences repeat themselves across countries.

The reported two-year HBR stock-out in Kenya coincides with healthcare devolution in the country; health service delivery is now the responsibility of the 47 county governments formed after the 2013 general election. Based on reported data from the Government of Kenya, HBR procurement and distribution in 2014 and 2015 remained a responsibility of the Ministry of Health at the national level, but it is possible that procurement of the HBR, and perhaps other recording tools, "fell between the cracks" in the changing landscape of healthcare/medical supplies and commodities and as well as the financial flows supporting them. Recent immunization programme reports highlight reductions in funding and delays in securing funding for vaccines and it is reasonable to presume recording tools are similarly impacted [5]. It is understandable that it will take time for county governments in Kenya to improve their capacity to manage such supply chain systems on their own or for alternate models to develop; however, further attention is needed to provide structure for the HBR supply chain management in order to resolve the two-year HBR stock-out problems.

The number of countries who either did not report or did not have data on whether there was a HBR stock-out is also concerning. Countries have been encouraged to include HBR availability among the programme performance indicators [6]. The absence of information on whether HBR stock-outs occur in a country may serve as a signal of more broad problems in a country's recording and reporting system, not unlike the situation with stock-outs of vaccines and injection supplies. It is important to monitor the quantity of HBRs available at the operational, district, provincial and national levels and to accurately forecast needs for home-based records to prevent stock-outs as well as unnecessary overstocks [1]. The importance of monitoring HBR availability and distribution is of particular importance in settings where more than one governmental department is involved in recording and reporting tool development/update processes as well as maintenance and management. As suggested above, inter-departmental communication and coordination failures have been previously cited though the frequency of the problem is unknown (and perhaps irrelevant).

It is important to note that the data reported here only reflect information regarding stock-outs at national level. HBR stock-outs at the district level may occur either in the presence or absence of stock-outs at national level. Unfortunately, the questions asked of countries for the 2014 and 2015 periods did not ask about HBR stock-outs at this level, which is an important limitation as highlighted by others [7] with regards to other commodities. Stock-outs may also occur in the private sector and are not included. The reported data also do not provide any indication of the duration of the HBR stock-out. Prior results ob- 
served national HBR stock-out durations on average of seven months at the national level and of six months at district level [1]. Also, our ability to tease out countries that chose not to report from those who did not have data on HBR stock-outs is limited here. Beginning with the 2017 data collection cycle for 2016 immunization system performance data, this issue will be addressed directly.

Our understanding of the root causes behind HBR stock-outs is currently lagging behind but it is necessary in order to develop sustainable solutions for both the near and long-term. Prior work [1] hypothesized complex HBR financing and coordination/management settings, such as when the responsibility of HBR procurement is a shared responsibility of the national immunization programme and another department (e.g., nutrition) and heavy dependence on external funding with rapid withdrawal of funding in the absence of dedicated budget lines in costed multi-year plans for HBRs as potential contributing factors. In other instances, simple neglect of the records as a priority against a backdrop of suboptimal tracking systems for durable commodities may explain much of the problem. Further operational research is needed to understand the viability and potential (dis-)advantages of bundling HBRs with other vaccination delivery commodities as mechanism to reduce the risk of such stock-outs. But, this is only one possible solution of many that might be explored.

Home-based records offer a simple and relatively inexpensive means to foster coordination and continuity of immunization service delivery while facilitating communication, promoting childhood immunization, educating caregivers about their child's immunization status and stimulating demand for services that complements facility-based recording practices. However, in order to meet these ends, first-and-foremost, a well-designed, durable HBR must be readily available in the right quantity, in the right place at the right time. HBR stock-outs, as with stock-outs of other vaccine delivery supplies, are avoidable given appropriate attention amongst the other priority areas of vaccine delivery. National immunization programmes, and the development partners supporting them, are encouraged to include monitoring HBR availability and adoption among their programme indicators, to raise their awareness of the challenges surrounding HBRs in their context and to leverage existing investment opportunities [8] for exploring sustainable solutions to correct deficiencies moving forward.

\section{Authors' Disclaimer}

The findings and views expressed herein are those of the authors alone and do not necessarily reflect those of their respective institutions.

\section{Competing Interests}

The authors have declared that no competing interests exist in conjunction with this work.

\section{Financial Disclosure}

MDG completed this work under her duties as an employee of the World Health 
Organization, Geneva, Switzerland. DWB completed this work as an employee of the Brown Consulting Group International, LLC under a professional services consultancy contract with the Bill and Melinda Gates Foundation. The authors received no specific funding for this work.

\section{Author Contributions}

MGD was involved in the collection of the data and final review and editing of the manuscript. DWB prepared the manuscript and was involved in the final review and editing of the manuscript.

\section{References}

[1] Young, S.L., Gacic-Dobo, M. and Brown, D.W. (2015) Results from a Survey of National Immunization Programmes on Home-Based Vaccination Record Practices in 2013. International Health, 7, 247-255. https://doi.org/10.1093/inthealth/ihv014

[2] United Nations Human Rights Office of the High Commissioner (1990) Convention on the Rights of the Child.

http://www.ohchr.org/Documents/ProfessionalInterest/crc.pdf

[3] World Health Organization. Global Vaccine Action Plan 2011-2020.

http://www.who.int/immunization/global_vaccine_action_plan/GVAP_doc_2011_2 020/en/

[4] Maternal and Child Health Integrated Program and US Agency for International Development. Bottlenecks and Breakthroughs: Lessons Learned from New Vaccine Introductions in Low-Resource Countries, 2008 to 2013. http://jsi.com/JSIInternet/Inc/Common/_download_pub.cfm?id=15580\&lid=3

[5] Gavi, the Vaccine Alliance. Joint Appraisal Report, Kenya (August 2015). http://www.gavi.org/country/kenya/documents/jas/joint-appraisal-kenya-2015/

[6] Brown, D.W. and Gacic-Dobo, M. (2015) Home-Based Record Prevalence among Children Aged 12 - 23 Months from 180 Demographic and Health Surveys. Vaccine, 33, 2584-2593. https://doi.org/10.1016/j.vaccine.2015.03.101

[7] Wagenaar, B.H., Gimbel, S., Hoek, R., Pfeiffer, J., Michel, C., Manuel, J.L., Cuembelo, F., Quembo, T., Afonso, P., Gloyd, S. and Sherr, K. (2014) Stock-Outs of Essential Health Products in Mozambique-Longitudinal Analyses from 2011 to 2013. Tropical Medicine and International Health, 19, 791-801. https://doi.org/10.1111/tmi.12314

[8] Hasman, A., Rapp, A. and Brown, D.W. (2016) Revitalizing the Home-Based Record: Reflections from an Innovative South-South Exchange for Optimizing the Quality, Availability and Use of Home-Based Records in Immunization Systems. Vaccine, 34, 5697-5699. https://doi.org/10.1016/j.vaccine.2016.09.064 


\section{Annex}

${ }^{\star}$ Countries reporting national level HBR stock-outs in both 2014 and 2015 are shown in bold type below.

1) Countries reporting a national level HBR stock-out during 2014 WHO African Region

Botswana, Burundi, Cameroon, Chad, Democratic Republic of the Congo,

Equatorial Guinea, Gabon, Guinea-Bissau, Kenya, Niger, Togo

WHO Region of the Americas

Argentina, Belize, Dominican Republic, Venezuela

WHO Eastern Mediterranean Region

\section{Somalia}

WHO Western Pacific Region

Lao People's Democratic Republic, Tuvalu, Vanuatu

2) Countries reporting a national level HBR stock-out during 2015 WHO African Region

Botswana, Cameroon, Central African Republic, Chad, Democratic Republic of the Congo, Equatorial Guinea, Ghana, Guinea-Bissau, Kenya, Malawi, Mauritania, Namibia

WHO Region of the Americas

Belize, Panama, Venezuela

WHO Eastern Mediterranean Region

Somalia

WHO South-East Asia Region

Timor-Leste

WHO Western Pacific Region

Cambodia, Kiribati, Lao People's Democratic Republic, Philippines, Solomon Islands

Submit or recommend next manuscript to SCIRP and we will provide best service for you:

Accepting pre-submission inquiries through Email, Facebook, LinkedIn, Twitter, etc. A wide selection of journals (inclusive of 9 subjects, more than 200 journals)

Providing 24-hour high-quality service

User-friendly online submission system

Fair and swift peer-review system

Efficient typesetting and proofreading procedure

Display of the result of downloads and visits, as well as the number of cited articles

Maximum dissemination of your research work

Submit your manuscript at: http://papersubmission.scirp.org/

Or contact wjv@scirp.org 